\title{
ON AN INEQUALITY OF MEAN CURVATURES OF HIGHER DEGREE ${ }^{1}$
}

\author{
BY BANG-YEN CHEN
}

Communicated by S. Sternberg, June 26, 1970

1. Introduction. Let $x: M \rightarrow E^{n+N}$ be an immersion of an $n$-dimensional closed manifold $M$ immersed in a euclidean space $E^{n+N}$ of dimension $n+N$. Let $B_{v}$ be the bundle of unit normal vectors of $x(M)$ so that a point of $B_{v}$ is a pair $(p, e)$, where $e$ is a unit normal vector to $x(M)$ at $x(p)$. Then $B_{v}$ is a bundle of $(N-1)$-dimensional spheres over $M$ and is a manifold of dimension $n+N-1$. Let $d V$ be the volume element of $M$. There is a differential form $d \sigma$ of degree $N-1$ on $B_{v}$ such that its restriction to a fibre is the volume element of the sphere of unit normal vectors at a point $p \in M$; then $d \sigma \wedge d V$ is the volume element of $B_{v}$. For each $(p, e) \in B_{v}$, there corresponds a symmetric linear transformation $A(p, e)$ of the tangent space $T_{p}(M)$ of $M$ at $p$, called the second fundamental torm at $(p, e)$. The eigenvalues $k_{1}(p, e), \cdots, k_{n}(p, e)$, of the second fundamental form $A(p, e)$ are called the principal curvatures at $(p, e)$. The $i$ th mean curvature $K_{i}(p, e), i=1,2, \cdots, n$, are defined by the elementary symmetric functions as follows:

$$
\left(\begin{array}{l}
n \\
i
\end{array}\right) K_{i}(p, e)=\sum k_{1}(p, e) \cdots k_{i}(p, e), \quad i=1,2, \cdots, n,
$$

where $\left(\begin{array}{l}n \\ i\end{array}\right)=n ! / i !(n-i) !$

We call the integral $K_{i}^{*}(p)=\int\left|K_{i}(p, e)\right|^{n / i} d \sigma$ over the sphere of unit normal vectors at $x(p)$, the $i$ th total absolute curvature of the immersion $x$ at $p$, and we define as the ith total absolute curvature of $M$ itself the integral $\int_{M} K_{i}^{*}(p) d V$.

In this note, I would like to announce the following results:

THEOREM 1. Let $x: M \rightarrow E^{n+N}$ be an immersion of a closed manifold of dimension $n$ into $E^{n+N}$. Then we have the follawing inequality:

AMS 1969 subject classifications. Primary 5374, 5390; Secondary 5345, 5399.

Key words and phrases. Closed submanifold, normal bundle, second fundamental form, $i$ th mean curvature, hypersphere.

1 This paper was presented to the Midwest Regional Conference in Differential Geometry at East Lansing, June 18, 1970. 


$$
\int_{M} K_{i}^{*}(p) d V \geqq 2 c_{n+N-1}, \quad i=1,2, \cdots, n,
$$

where $c_{n+N-1}$ denotes the area of the unit $(n+N-1)$-sphere. The equality sign of (2) holds when and only when $M$ is imbedded as a hypersphere in an $(n+1)$-dimensional linear subspace of $E^{n+N}$ if $i<n$, and as a convex hypersurface in an $(n+1)$-dimensional linear subspace of $E^{n+N}$ if $i=n$.

REMARK. If $i=n$, then this theorem is the well-known FenchelBorsuk-Chern-Lashof's theorem [1], [4], [5], and if $i=1$, this theorem was proved by Willmore-Chen [2], [3], [6].

THEOREM 2. Under the hypothesis of Theorem 1, if the mean curvature normal $H(p)$ has constant length; $|H(p)|=\left(c_{n} / v(M)\right)^{1 / n}$, then $M$ is immersed as a hypersphere with radius $\left(v(M) / c_{n}\right)^{1 / n}$ in an $(n+1)$ dimensional linear subspace of $E^{n+N}$, where $v(M)$ denotes the volume of $M$.

THEOREM 3. Under the hypothesis of Theorem 1, if $N=1$ and $\left|K_{i}(p, e)\right|^{n}=\left(c_{n} / v(M)\right)^{i}$, then $M$ is immersed as a hypersphere with radius $\left(v(M) / c_{n}\right)^{1 / n}$.

Theorem 2 and Theorem 3 follow immediately from Theorem 1.

2. Sketch of the proof of Theorem 1. Fix a unit vector $e \in S_{0}^{n+N-1}, S_{0}^{n+N-1}$ the unit hypersphere in $E^{n+N}$, the scalar product $e \cdot x(p)$ as a continuous function on $M$ has at least one maximum and one minimum, say $q$ and $q^{\prime}$, respectively. Since at $(q, e)$ and $\left(q^{\prime}, e\right)$, the second fundamental form $A(p, e)$ is semidefinite. Let $d \Sigma$ denote the volume element of $S_{0}^{n+N-1}$ and define

$$
\tilde{v}: B_{v} \rightarrow S_{0}^{n+N-1}
$$

by $\delta(p, e)=e$. Then we have

$$
\tilde{v}^{*} d \Sigma=K_{n}(p, e) d V \wedge d \sigma .
$$

Therefore, by Sard's theorem, we know that for almost all $e$ in $S_{0}^{n+N-1}$, the second fundamental form $A(p, e)$ is definite for extreme points of the function $e \cdot x(p)$ on $M$. Hence, if we let $U^{*}$ denote the set of all elements $(p, e)$ in $B_{v}$ such that $A(p, e)$ is definite, then we have

$$
\int_{U^{*}}\left|K_{n}(p, e)\right| d \sigma \wedge d V \geqq 2 c_{n+N-1} .
$$

On the other hand, we have 


$$
\begin{aligned}
\left|K_{1}(p, e)\right|^{n} & \geqq\left|K_{2}(p, e)\right|^{n / 2} \geqq \cdots \geqq\left|K_{i}(p, e)\right|^{n / i} \\
& \geqq \cdots \geqq\left|K_{n}(p, e)\right|,
\end{aligned}
$$

on $U^{*}$. Therefore, by (5) and (6), we get

$$
\int_{M} K_{i}^{*}(p) d V \geqq \int_{U^{*}}\left|K_{i}(p, e)\right|^{n / i} d \sigma \wedge d V \geqq 2 c_{n+N-1} .
$$

This proves (2).

Furthermore, if the equality sign of (2) holds, then we have

$$
\left|K_{i}(p, e)\right|^{n}=\left|K_{n}(p, e)\right|^{i} \text {, on } U^{*}
$$

and

$$
K_{i}(p, e)=0, \quad \text { on } B_{v}-U^{*} .
$$

By using (8), (9) and the continuity of $K_{i}(p, e)$ on $M$, we can prove that $B_{v}-U^{*}=\left\{(p, e) \in B_{v}: k_{1}(p, e)=\cdots=k_{n}(p, e)=0\right\}$. Using these facts and Theorem 3 of [4], we can prove the remaining part of theorem without difficulty.

\section{REFERENCES}

1. K. Borsuk, Sur la courbure totale des courbes fermées, Ann. Soc. Polon. Math. 20 (1947), 251-265. MR 10, 60.

2. B. -Y. Chen, On an inequality of T. J. Willmore, Proc. Amer. Math. Soc. (to appear).

3. - On the total curvature of immersed manifolds. I: An inequality of FenchelBorsuk-Willmore (to appear).

4. S.-S. Chern and R. K. Lashof, On the total curvature of immersed manifolds, Amer. J. Math. 79 (1957), 306-318. MR 18, 927.

5. W. Fenchel, Über Krümmung und Windung geschlossener Raumkurven. Math. Ann. 101 (1929), 238-252.

6. T. J. Willmore, Mean curvature of immersed surfaces, An. Ști. Univ. "Al. I. Cuza” Iasi. Secț. I a Mat. 14 (1968), 99-103. MR 38 \#6496.

Michigan State University, East Lansing, Michigan 48823 